\title{
Capsaicina al 8\% para controlar el dolor neuropático periférico: una estrategia basada en casos clínicos
}

\author{
A. Bhaskar' ${ }^{1}$ J. Nadstawek², E. J. Viel ${ }^{3}$, M. Buonocore ${ }^{4}$, J. M. López Millán ${ }^{5}$ y H. Jaeger ${ }^{6}$ \\ ${ }^{I}$ Especialista en Medicina del Dolor. Medicina del Dolor y Anestesia. The Christie NHS Foundation Trust \\ Withington Manchester Reino Unido. ${ }^{2} J e f e$ de la Clínica del Dolor y la Unidad de Tratamiento del Dolor \\ Postoperatorio. Departamento de Anestesiología y Cuidados Críticos. Universidad Rheinische Friedrich-Wilhelms \\ de Bonn. Alemania. ${ }^{3}$ Jefe del Servicio de Tratamiento del Dolor. Clínica del Dolor. Hospital Universitario de \\ Caremeau. Francia. ${ }^{4}$ Jefe de la Unidad de Neurofisiología Clínica. Fundación Salvatore Maugeri. Instituto \\ Científico de Pavía. Pavía. Italia. ${ }^{5}$ Jefe de Anestesiología y de la Unidad Integral de Cuidados Críticos y del Dolor. \\ Hospital Universitario Virgen Macarena. Sevilla. España. ${ }^{6}$ Especialista en Dolor y Anestesiólogo. Centro para el \\ Tratamiento y el Estudio del Dolor de Hamburgo. Praxis für Schmerz- und Psychotherapie. Hamburgo. Alemania
}

Bhaskar A, Nadstawek J, Viel EJ, Buonocore M, López Millán JM, Jaeger H. Capsaicina al 8\% para controlar el dolor neuropático periférico: una estrategia basada en casos clínicos. Rev Soc Esp Dolor 2013; 20(1): 25-33.

\begin{abstract}
The Astellas peer-to-peer meeting took place in Milan on the evening of Tuesday 28 August, 18:00-20:00. Approximately 150 invited pain physicians from across Europe, all with some knowledge or experience of using the high-dose capsaicin patch to treat peripheral neuropathic pain (NP), attended the meeting to hear an expert European faculty of practising physicians present different case studies involving treatment with high-dose capsaicin. The objective of the meeting was to share real-world experience in the use of high-dose capsaicin for the management of NP in an interactive format to provide delegates with the opportunity to liaise with physicians who have direct, handson experience of high-dose capsaicin. Interactivity was a key component of the meeting and iPad technology was utilised to encourage discussions between the faculty and the delegates. The ThoughtSort application enabled both the faculty to ask delegates questions related to their case studies and the delegates to ask the faculty questions throughout their presentations.

The meeting was chaired by Dr Arun Bhaskar from the UK. $\mathrm{He}$ opened the meeting by welcoming the delegates, introducing the faculty, outlining the objectives of the meeting and performing a demonstration of ThoughtSort. The delegates had to work in groups to answer questions via ThoughtSort, using one iPad between four and five delegates. Details of the case studies
\end{abstract}

that were presented at the meeting and summarised within this report are the personal opinions of the faculty members.

\section{RESUMEN}

La reunión multidisciplinar organizada por Astellas tuvo lugar en Milán el 28 de agosto de 2012. Alrededor de 150 médicos invitados, especializados en el dolor, procedentes de toda Europa y con conocimientos o experiencia en el uso de los parches de capsaicina al $8 \%$ para tratar el dolor neuropático (DN) periférico, asistieron a la reunión para escuchar la presentación de distintos casos clínicos tratados con el parche de capsaicina al 8\%, por parte de un grupo de ponentes europeos expertos en la clínica del dolor. El objetivo de la reunión era compartir experiencia clínica real sobre el uso del parche de capsaicina al 8\% para controlar el DN siguiendo un formato interactivo y dando a los asistentes la oportunidad de intercambiar experiencia práctica directa con el uso de este medicamento.

La reunión estuvo presidida por el Dr. Arun Bhaskar del Reino Unido. Este inauguró la reunión dando la bienvenida a los delegados, presentando a los ponentes, resumiendo los objetivos de la reunión y realizando una demostración del ThoughtSort, el software que por grupos a través de un iPad, hizo posible que todos los participantes de la sesión pudiesen interactuar. Los detalles de los casos clínicos que se presentaron en la reunión y que se resumen en este artículo responden a las opiniones personales de los ponentes.

Palabras clave: Dolor neuropático. Capsaicina. Casos clínicos. Ipad, ThoughtSort. 
Rev. Soc. Esp. del Dolor, Vol. 20, N. ${ }^{\circ}$ 1, Enero-Febrero 2013

\section{TRATAMIENTO DE LA NEURALGIA POST-HERPÉTICA CON EL PARCHE DE CAPSAICINA AL 8\%: CASO CLÍNICO}

El profesor Joachim Nadstawek de Alemania presentó el caso clínico de una paciente con neuralgia post-herpética (NPH). A esta mujer de 68 años de edad le habían diagnosticado una NPH en marzo de 2012, después de un episodio de herpes zóster que había afectado a la raíz T4 en diciembre de 2011. El dolor era constante, presentaba exacerbaciones y llegaba a los 8 puntos de intensidad. Su marido, que era neurólogo, le recetó varios medicamentos y la paciente tomaba pregabalina, amitriptilina y metamizol cuando acudió a la clínica del dolor en Bonn en mayo de 2012. La puntuación de dolor era de $6 \mathrm{y}$, en consecuencia, la paciente no quería reducir la dosis de pregabalina, pues la proporcionaba un cierto alivio. El profesor Nadstawek le prescribió un parche de lidocaína y le retiró la amitriptilina y el metamizol. Seis semanas después, la paciente refería seguir padeciendo un dolor de 6 puntos pero con menos exacerbaciones y menos dolores punzantes. También había podido reanudar ciertas actividades de jardinería.

Llegado a este punto de la presentación, el profesor Nadstawek pidió a los delegados que votaran cuál sería el tratamiento que elegirían a continuación. La mayoría de los grupos de delegados dijeron que aplicarían el parche de capsaicina al $8 \%$ manteniendo la pregabalina (Tabla I).

Después de explicarle a la paciente el tratamiento con el parche de capsaicina al $8 \%$, sus ventajas y sus desventajas, el profesor Nadstawek había optado por esta opción terapéutica. La paciente no quería recibir más medicación sistémica que pudiera afectar a sus capacidades cognitivas. Dos semanas después de la consulta, el 23 de julio de 2012, con una puntuación de dolor de 7, a la paciente se le aplicó un parche de capsaicina al $8 \%$ durante 60 minutos, después de haber sido pretratada con crema de lidocaína durante 60 minutos. El efecto pareció ser inmediato; la paciente refirió

TABLA I. ¿QUÉ TRATAMIENTO ELEGIRÍAN A CONTINUACIÓN? $\mathrm{N}=23$. ISRN $=$ INHIBIDOR SELECTIVO DE LA RECAPTACIÓN DE NORADRENALINA.

Continuar con el mismo tratamiento y hacer otro control al cabo de 1 mes

$0 \%$

Añadir un opioide a la pauta de tratamiento

$14 \%$

Tratar con el parche de capsaicina al

$8 \%$ mientras se sigue administrando pregabalina

Añadir un ISRN al tratamiento

$0 \%$

Probar con un ISRN; si no resulta, probar con un opioide; si no resulta, tratar con capsaicina al $8 \%$ que la puntuación de dolor era de 1-2 al darle el alta. En la primera visita de seguimiento 1 semana después, la paciente estaba feliz, sin síntomas y con una puntuación de dolor de 0-1. Pasados otros 7 días, la paciente no tenía dolor y refería que ya podía hacer vida normal.

Los delegados votaron después qué medidas tomarían en el futuro respecto a esta paciente; el $84 \%$ de los grupos dijeron que repetirían el tratamiento con el parche de capsaicina y reducirían la pregabalina (Tabla II).

Aunque se necesitan más estudios para comparar las eficacias de la capsaicina al $8 \%$ y la pregabalina, así como estudios farmacoeconómicos, el profesor Nadstawek aconsejó no plantearse el uso del parche de capsaicina al $8 \%$ como última opción de tratamiento en los pacientes con NPH.

\section{Coloquio}

- ¿Por qué no se subió la dosis de pregabalina a $2 \times$ $300 \mathrm{mg}$ ? Esa es una dosis muy alta de pregabalina y la paciente expresó su decidido deseo de conservar la claridad mental y poder seguir leyendo. A la paciente le preocupaban los efectos secundarios de una dosis demasiado alta y por ello se empleó el parche de capsaicina al $8 \%$ añadido a la pauta ya existente de pregabalina.

- ¿Cuál es su experiencia respecto a la duración del tratamiento con capsaicina? El profesor Nadstawek opinó que, si existe una buena respuesta al tratamiento, y dado que el parche de capsaicina al $8 \%$ tiene efectos secundarios mínimos, el tratamiento debe repetirse al cabo de 3 meses tal como se especifica en la ficha técnica.

\section{DOLOR CRÓNICO DE MUÑÓN Y MIEMBRO FANTASMA EN UN AMPUTADO: EFICACIA DEL PARCHE DE CAPSAICINA AL $8 \%$ Y JUSTIFICACIÓN FARMACOECONÓMICA DE SU EMPLEO}

El caso clínico presentado por el profesor Eric Viel de Francia trata de un arquitecto varón de 64 años con dolor

TABLA II. ¿QUÉ MEDIDAS TOMARÍAN EN EL FUTURO? $\mathrm{N}=25$.

Repetir el tratamiento con parches de capsaicina al cabo de 3 meses sin cambiar $0 \%$ la medicación adicional

Repetir el tratamiento con parches de capsaicina $8 \%$ y reducir la pregabalina

$88 \%$

Repetir el tratamiento con parches de capsaicina 8\% retirar la pregabalina 
crónico de muñón y de miembro fantasma a raíz de una lesión de carpintería que llevó a la amputación de los dedos medio, anular y meñique de la mano izquierda. El paciente se sometió a la amputación en abril de 2010 y acudió a la clínica del dolor en junio de 2011. En ese momento, el paciente tenía dolor de muñón constante, con una puntuación de 7/10 en el cuestionario DN4. El dolor lo describía en forma de quemazón, descargas eléctricas, hormigueo, entumecimiento, picor, hiposensibilidad táctil y alodinia. El paciente también refería varios episodios de dolor intercurrente a la hora, de unos 10 minutos de duración cada uno. Estos alcanzaban el 70 en una EVA de 0-100. El paciente también padecía dolor de miembro fantasma al estimular los muñones de los dedos.

El paciente había recibido tratamiento con amitriptilina, gabapentina y clonazepam, pero los tres se suspendieron por poco eficaces. Tampoco obtuvo alivio alguno de la aplicación diaria de parches de lidocaína durante 12 horas a lo largo de 3 meses. El tratamiento con pregabalina y tramadol redujo en un $30 \%$ la intensidad del dolor de muñón, pero no alivió el dolor de miembro fantasma, asociándose esta combinación a efectos adversos. El paciente estaba "totalmente destrozado", había engordado y tenía la libido baja.

La estrategia terapéutica propuesta por el profesor Viel consistió en proseguir con la pregabalina diaria (200 mg dos veces al día), instaurar la duloxetina (60 mg al día antes de acostarse) y recibir tratamiento con parches de capsaicina al 8\%. Al paciente se le aplicó el primer parche en junio de 2011 y el segundo en julio de 2011, a pesar de que esta no es la posología que se especifica en la ficha técnica, que explica que el tratamiento puede repetirse cada 90 días en función de la persistencia o la recidiva del dolor. En agosto de 2011 ya se había resuelto el dolor de miembro fantasma pero el de muñón seguía igual. El paciente seguía tomando la misma pauta de medicación sistémica. En octubre de 2011 se le aplicó el tercer parche. Un mes después la intensidad del dolor de muñón se había reducido en un $40 \%$ y este solo persistía en los dedos anular y meñique. La dosis de pregabalina se redujo a $75 \mathrm{mg}$ dos veces al día y el paciente tomó la decisión de suspender la duloxetina a causa de la fatiga y la reducción del impulso sexual. Durante un periodo de 5 meses en que estuvo de viaje, el paciente decidió seguir con la pregabalina en dosis de 50 mg una vez al día pero, en junio de 2012, el dolor de muñón había reaparecido en los tres dedos. El dolor del miembro fantasma no recidivó. En ese momento, el paciente reanudó la pregabalina en dosis de $50 \mathrm{mg}$ dos veces al día y se le aplicó un cuarto parche. El dolor se resolvió totalmente 2 meses después del tratamiento (agosto de 2012) y se programó la retirada gradual de la pregabalina.

El profesor Viel explicó la justificación económica del uso del parche de capsaicina al $8 \%$, presentando datos procedentes de un estudio preliminar de 31 pacientes. Los
TABLA III. DE MEDIA, ¿QUÉ REDUCCIÓN OBSERVAN EN EL USO DE MEDICAMENTOS CONCOMITANTES PARA EL DOLOR NEUROPÁTICO EN LOS PACIENTES QUE HAN TRATADO CON EL PARCHE DE CAPSAICINA AL $8 \% ? \mathrm{~N}=30$.

\begin{tabular}{lc}
\hline $0-24 \%$ & $17 \%$ \\
$25-49 \%$ & $40 \%$ \\
$50-74 \%$ & $37 \%$ \\
$75-100 \%$ & $3 \%$ \\
Sin experiencia & $3 \%$ \\
\hline
\end{tabular}

datos de este estudio mostraban que el 16\% de los pacientes habían suspendido todos los analgésicos y el $61 \%$ habían reducido la medicación sistémica. Se estimó que esto suponía un ahorro de entre $250 €$ y $340 €$ por paciente en un periodo de 90 días.

La mayoría de los grupos de asistentes también habían observado descensos de la medicación concomitante para el DN después de recibir tratamiento con el parche de capsaicina al 8\% (Tabla III).

El paciente en este caso experimentó una mejoría de la calidad de vida después del tratamiento con el parche de capsaicina al $8 \%$. Además del alivio del dolor y de ver resuelto el dolor de miembro fantasma, el paciente también vio aliviados los efectos adversos causados por la medicación sistémica, aumentando los niveles de energía y la libido.

Se preguntó a los asistentes qué mejorías de la calidad de vida habían observado en los pacientes. Muchos dijeron que habían visto mejorar el sueño, pero en otros casos también la actividad mental y la funcionalidad, permitiendo la vuelta al trabajo y la capacidad de poder vestirse correctamente y usar zapatos de nuevo (Fig. 1).

La mayoría de los grupos de delegados pretratan habitualmente a sus pacientes con un anestésico local como la EMLA o la lidocaína (Tabla IV). Sin embargo, existe entre los médicos un encendido debate sobre el pretratamiento, sobre todo porque la crema de lidocaína no siempre funciona y existen muchas otras opciones.

\section{Coloquio}

- ¿Cómo explica el efecto positivo sobre el dolor de miembro fantasma? En los nervios seccionados existe una mayor concentración de receptores TRPV1 (Transient Receptor Potential Vanilloid 1). Por tanto, es posible que en el muñón de una amputación haya una concentración alta de receptores TRPV1, lo que explicaría porqué en estos casos actúa positivamente el parche de capsaicina al $8 \%$. 


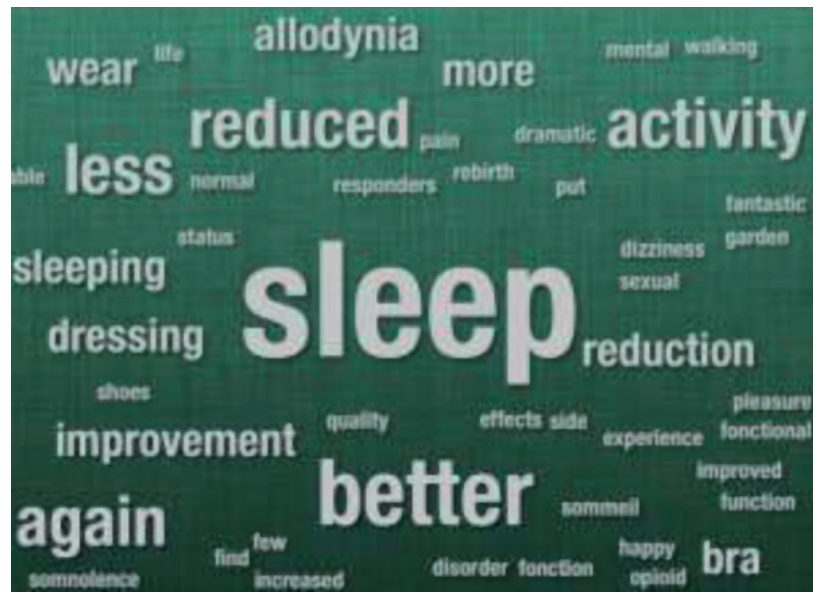

Fig. 1. ¿Qué mejorías de la calidad de vida han observado en sus pacientes? $\mathrm{N}=31$. Se pidió a los asistentes que respondieran con una o dos palabras para poder mostrar los resultados en forma de nube de palabras. El tamaño de cada palabra de la nube es directamente proporcional al número de veces que se ha utilizado.

- ¿Cómo decide el momento de tratar de nuevo al paciente? El profesor Viel no espera 3 meses con sus pacientes. En su experiencia, la zona con DN se reduce después de la primera aplicación del parche pero no desaparece del todo. En consecuencia, él volvería a aplicar el parche pero solo sobre el área reducida.

- ¿En qué otras etiologías le ha funcionado bien Qutenza? En otras etiologías como la NPH, las cicatrices postquirúrgicas y la neuropatía inducida por quimioterapia.

\section{SÍNDROME DE DOLOR REGIONAL COMPLEJO (TIPO II): ¿PUEDE TRATARSE CON CAPSAICINA AL 8\%?}

El Dr. Michelangelo Buonocore, de Italia, presentó el caso de un paciente con síndrome de dolor regional complejo (SDRC) de tipo II y preguntó si esta enfermedad podría tratarse con el parche de capsaicina al $8 \%$. Al paciente le habían diagnosticado un síndrome del túnel carpiano en la mano derecha en 2009 y se sometió a una neurólisis quirúrgica, primero del nervio mediano derecho y después del izquierdo. Hubo cierta mejoría de las parestesias en ambas manos y de la velocidad de conducción

TABLA IV. ¿PRETRATAN HABITUALMENTE CON UN ANESTÉSICO TÓPICO? N = 30 .

\begin{tabular}{ll}
\hline $\mathrm{Si}$ & $83 \%$ \\
$\mathrm{No}$ & $17 \%$ \\
\hline
\end{tabular}

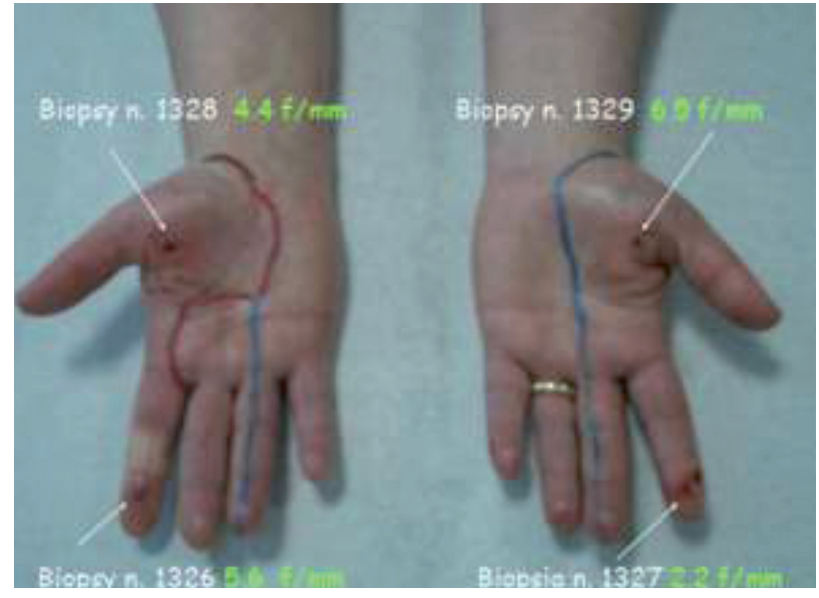

Fig. 2. Localización de las biopsias neurodiagnósticas de piel.

distal en ambos nervios, pero al paciente le diagnosticaron un SDRC en junio de 2010.

El paciente acudió a la unidad del dolor en febrero de 2011 con dolor espontáneo intenso en ambas manos, principalmente en la derecha, asociado a parestesias, alodinia táctil y rigidez. El paciente era supuestamente resistente al tramadol, la oxicodona, la carbamazepina y la duloxetina, y tomaba $75 \mathrm{mg} /$ día de amitriptilina y $150 \mathrm{mg} /$ día de pregabalina, siendo la analgesia poco satisfactoria. La exploración física detectó alodinia mecánica dinámica, hiperalgesia a los pinchazos acompañada de hipoestesia térmica, eritema e hiperhidrosis. Se añadió al tratamiento prednisolona en dosis de $25 \mathrm{mg}$ /día durante 3 semanas para conocer la función patogénica de la inflamación y posiblemente mejorar la eficacia, pero el paciente no reflejó cambio alguno en la escala Patient Global Impression of Change (PGIC) y se le retiró la prednisolona por ineficaz.

En vista de lo observado en la exploración física, en abril de 2011 se le realizaron cuatro biopsias neurodiagnósticas de piel en ambas manos (Fig. 2). Los resultados de las biopsias mostraban una reducción de la densidad de fibras nerviosas en la epidermis de las yemas de los dedos de la mano izquierda.

Al paciente se le aplicaron tres parches de capsaicina al $8 \%$ a intervalos de 12 semanas. En la semana 1, posterior al primer tratamiento, la PGIC había mejorado mínimamente, pero al cumplirse la semana 12, el paciente refería una puntuación de 4 en esta escala. Después del segundo tratamiento, la mejoría era mínima en la semana 2 y los síntomas habían empeorado mínimamente en la 12. Después del tercer parche se produjo una mejoría gradual hasta que el paciente pudo volver al trabajo en la semana 12, con una importante mejoría de la PGIC.

Después de aplicar el parche de capsaicina se observaron distintos patrones de eritema; el eritema era menor en 
TABLA V. TENIENDO EN CUENTA LA ANATOMÍA NORMAL Y LAS CARACTERÍSTICAS CLÍNICAS DEL PACIENTE DESCRITO, ¿CREEN QUE LA DISOCIACIÓN SE DEBE FUNDAMENTALMENTE A...? N = 21 .

\begin{tabular}{lc}
\hline $\begin{array}{l}\text { Menor densidad de inervación en la piel } \\
\text { glabra de... }\end{array}$ & $5 \%$ \\
Menos fibras nerviosas con expresión de & $40 \%$ \\
TRPV1 en el nervio mediano... & $55 \%$ \\
\hline
\end{tabular}

la superficie palmar de las manos. La mitad de los grupos de asistentes pensaron que la causa de esta diferencia era tanto por la menor densidad de inervación de la piel glabra en los sujetos normales (en comparación con la piel pilosa) como por el menor número de fibras nerviosas con expresión de TRPV1 en el territorio cutáneo de la lesión del nervio mediano (Tabla V). Es probable que intervengan los dos mecanismos, pero lo importante es observar que la densidad de inervación epidérmica es menor en la piel glabra que en la pilosa.

El paciente presentó una clara reducción de la zona dolorosa entre la primera y la segunda aplicación. Este es un posible signo de reducción de los fenómenos de sensibilización central, pues la aparición de dichos fenómenos se debe a la acción periférica de las fibras C. Sin embargo, la mayoría de los grupos de asistentes (62\%) no pensaban que este fuera el mecanismo analgésico más importante de la capsaicina al 8\% (Tabla VI). Es importante recordar que, aunque la principal acción de la capsaicina se ejerce sobre las fibras periféricas, siempre que la periferia se bloquee, existe también la posibilidad de que también resulte bloqueado cualquier tipo de sensibilización central.

Dadas las amplias variaciones fenotípicas de los distintos pacientes con DN, se necesitan claramente herramientas capaces de predecir quién va a responder al tratamiento con dosis altas de capsaicina. Las investigaciones preliminares del Dr. Buonocore y sus colaboradores muestran resultados mejores del parche de capsaicina al $8 \%$ en los pacientes con sensibilidad normal al calor, dolor más superficial que profundo y dolor provocado más que espontáneo.

\section{Coloquio}

- ¿Se ha producido alguna reacción alérgica a Quten$z a$ ? No en la experiencia del Dr. Buonocore.

- ¿Por qué realizaría alguien una neurólisis del nervio mediano en un síndrome del túnel carpiano? No es una intervención habitual y el Dr. Buonocore no sabía la respuesta ya que la intervención se había llevado a cabo antes de que el paciente le fuera derivado.
TABLA VI. LA CAPSAICINA AL $8 \%$ ACTÚA SOLAMENTE SOBRE LOS NERVIOS PERIFÉRICOS Y NO TIENE NINGÚN EFECTO SOBRE EL DOLOR NEUROPÁTICO CENTRAL. LA REDUCCIÓN DE LA ZONA DOLOROSA OBSERVADA EN ESTE PACIENTE DESPUÉS DE APLICAR EL PARCHE INDICA UNA DISMINUCIÓN DE LA SENSIBILIZACIÓN CENTRAL. ¿CREEN USTEDES QUE LA REDUCCIÓN DE LA SENSIBILIZACIÓN CENTRAL ES EL MECANISMO ANALGÉSICO MÁS IMPORTANTE DEL PARCHE DE CAPSAICINA? $\mathrm{N}=29$.

\begin{tabular}{lc}
\hline Sí & $31 \%$ \\
No & $62 \%$ \\
No sé & $7 \%$ \\
\hline
\end{tabular}

\section{SÍNDROME POSMASTECTOMÍA TRATADO CON CAPSAICINA AL 8\%: CASO CLÍNICO}

El síndrome doloroso posterior a la mastectomía (SDPM) es un nuevo síndrome que afecta a las mujeres mastectomizadas debido a un cáncer de mama. Puede cursar con dolor de la mama fantasma, síndrome intercostobraquial y dolor neuromatoso. Los signos y síntomas del SDPM se observan en la axila, la cara medial del brazo, el pecho y la cicatriz quirúrgica, y consisten en dolor urente, dolor punzante, entumecimiento, parestesias y alodinia. El SDPM es una afección normalmente larga; el 52\% de las pacientes refieren su continuidad 9 años después de la cirugía. El SDPM también puede afectar negativamente a la calidad de vida; las pacientes con SDPM continuo tienen peor calidad de vida que aquellas en que el síndrome se ha resuelto. Las opciones terapéuticas del SDPM abarcan medicamentos sistémicos y tópicos para el DN, técnicas de neuromodulación y bloqueos nerviosos.

El caso de SDPM presentado por el Dr. José López Millán se refiere a una mujer de 55 años que había sufrido una mastectomía total en 2008 y se había sometido a una cirugía reconstructiva en 2011. La paciente había estado tomando amitriptilina, escitalopram, pregabalina, bromazepam, oxicodona, mirtazapina y exemestano. Además de tener reducida la movilidad del hombro izquierdo, la paciente presentaba también una serie de síntomas, como hormigueo, disestesia e hiperalgesia, en la cara interna del miembro superior izquierdo, desde el hombro hasta el codo. Tenía una puntuación EVA de 10/10, una DN4 de 6/10 y un índice LATTINEN de 16. La paciente presentaba asimismo efectos secundarios de la medicación sistémica, somnolencia, mareo y falta de concentración. Se le diagnosticó un síndrome intercostobraquial y se le aplicaron cuatro parches de capsaicina al 8\% a lo largo de un año.

Después de este periodo, la paciente tenía menos dolor, la zona de alodinia había disminuido considerablemente, 


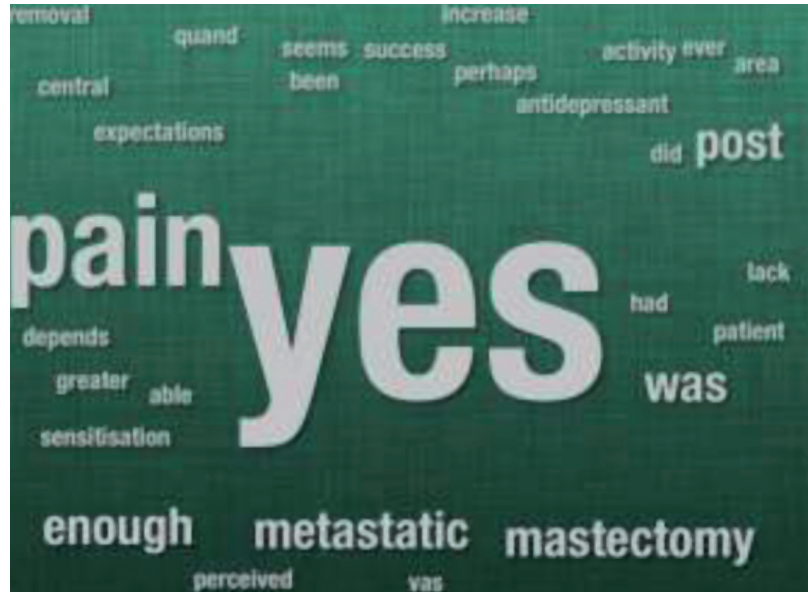

Fig. 3. Teniendo en cuenta las mejorías de las escalas EVA, DN4 y LATTINEN en este caso, ¿no podríamos esperar una mejor puntuación en la PGIC (Patient Global Impression of Change) después del tratamiento? $\mathrm{n}=28$. Muchas de las respuestas a esta pregunta tenían $\geq 2$ palabras, lo que ha dado lugar a una falsa representación de las respuestas de los delegados en la nube de palabras. Parece que la mayoría de los grupos de delegados respondieron que "sí", que hubieran esperado ver una mejor puntuación PGIC; sin embargo, al evaluar las respuestas completas, la puntuación PGIC no había sorprendido a la mayoría.

la movilidad del hombro había aumentado y la medicación sistémica se había reducido. La paciente tenía una EVA de 5/10, una DN4 de 3/10 y un índice LATTINEN de 14. La puntuación PGIC era también de 5 puntos. La paciente estaba ganando peso, mejoró su estado de ánimo y se encontraba parcialmente satisfecha con el tratamiento. La capsaicina en dosis altas puede usarse para tratar con éxito el SDPM, reduciendo la intensidad del dolor y las zonas de alodinia, sin efectos secundarios importantes ni durante el tratamiento ni después del mismo.

Se preguntó a los grupos de participantes si habrían esperado una mejor puntuación PGIC en vista de las mejorías de las puntuaciones e índices de dolor, comunicadas. En general, el 39\% de los grupos esperaban ver una mejor puntuación PGIC, mientras que al $43 \%$ no les sorprendía la peor puntuación en esta escala (Fig. 3). Los participantes comentaron que las mejorías nulas o solo mínimas de la actividad pueden afectar negativamente a la PGIC; además, la paciente podría haber esperado un mejor resultado y uno de los grupos comentó que todas las zonas de dolor que persisten parecen percibirse como una falta de éxito por parte del paciente. Se dijo también que la paciente no solo presentaba dolor secundario a la mastectomía sino también dolor metastásico en la zona de T12.

El Dr. López Millán comentó que, para esta paciente, el objetivo era aliviar el dolor, lo cual se consiguió.

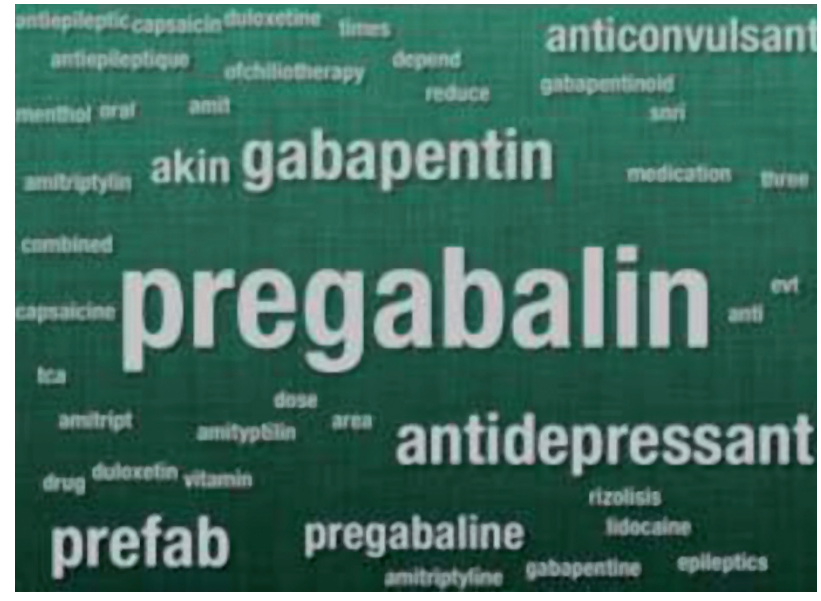

Fig. 4. La neuropatía inducida por quimioterapia puede ser otra causa de dolor neuropático en las pacientes que reciben este tipo de tratamiento a causa de un cáncer de mama. En caso de neuropatía inducida por quimioterapia, ¿cuál sería su primera línea de tratamiento? $\mathrm{N}=31$.

Sin embargo, la calidad de vida y las expectativas de las enfermas deben definirse junto con estas antes de que reciban tratamiento para poder ayudarlas a alcanzar las metas deseadas.

De los 31 grupos de participantes, la mayoría habría utilizado un anticonvulsivante o un antidepresivo (o una combinación de ambos) como tratamiento de primera línea en un caso de neuropatía inducida por quimioterapia (Fig. 4).

\section{Coloquio}

- ¿Se ha encontrado con fracasos del tratamiento con capsaicina? No en este caso. El fracaso terapéutico es un tema que hay que evaluar en función de las distintas indicaciones y situaciones ya que se pueden producir resultados diferentes, pudiendo resultar difícil la comparación entre pacientes; sin embargo, en los casos de dolor postquirúrgico se han observado muy buenos resultados con la capsaicina al $8 \%$.

- ¿Es más dolorosa la aplicación en esta zona? No, no más que en otras zonas. Se aplicó crema anestésica tópica (lidocaína al 5\%) antes del tratamiento y a la paciente le dimos antiinflamatorios no esteroideos (AINE), pero no se precisó más analgesia. Los opioides se usan solo a veces como rescate, pero no fueron necesarios en este caso.

- ¿En cuánto se redujo la medicación oral? Hubo una reducción gradual de los medicamentos episódicos pero el tratamiento basal siguió siendo el mismo. 


\section{CAPSAICINA AL 8\% COMO TRATAMIENTO DE LA NEUROPATÍA INDUCIDA POR QUIMIOTERAPIA: ESTRATEGIAS EFICACES DE TRATAMIENTO Y CONTROL DEL PACIENTE}

\section{El Dr. Arun Bhaskar, del Christie NHS Foundation Trust} del Reino Unido, presentó un caso de neuropatía inducida por quimioterapia (NIQ). La NIQ debuta como una neuropatía periférica de fibras pequeñas en manos y pies. La producen muchos de los quimioterápicos habituales, como los alcaloides de la vinca, los compuestos de platino, los taxanos y la talidomida. El quinto caso que se presentó y debatió fue el de una mujer de 54 años con cáncer de mama que había sido objeto de una tumorectomía y recibido ocho ciclos de oxaliplatino y trastuzumab. La paciente estaba en remisión pero había comenzado a presentar síntomas de NIQ después del cuarto ciclo de oxaliplatino, 20 meses antes de su derivación a la clínica del dolor para su tratamiento. La paciente presentaba pinchazos intensos y constantes en ambos pies, con frecuentes episodios de hormigueo, entumecimiento con sensaciones de descarga eléctrica, alodinia, hiperpatía, poca movilidad (la paciente no podía llevar zapatos) y alteración del sueño por ser molesto el contacto con la ropa de cama. La paciente ya había presentado previamente poca tolerancia a gabapentina, pregabalina, amitriptilina, morfina y oxicodona, y tomaba codeína y paracetamol. Se le trataron los dos pies con el parche de capsaicina al $8 \%$ durante 30 minutos. El tratamiento se toleró bien y no se precisó analgesia de rescate por molestias asociadas al mismo. A la paciente se le recomendó aplicar en la zona bolsas de frío en casa a demanda. Las pacientes que reciben tratamiento en los pies pueden sentir quemazón de inicio diferido tras la retirada del parche, a menudo comienza durante la primera noche después de la aplicación y puede durar hasta una semana.

Durante la primera conversación telefónica de seguimiento con la paciente, esta refirió que la quemazón se había iniciado 6 horas después del tratamiento. Se recomendó la aplicación de frío para aliviar esta molestia. En la consulta de seguimiento al cabo de 4 semanas, la paciente estaba muy preocupada por el dolor que había padecido a raíz del tratamiento, afirmando que había sido "el peor dolor que había sentido jamás" y que "había sufrido demasiado durante tres días". Sin embargo, al preguntarle sobre el dolor en curso, la paciente refirió que no sentía dolor alguno, que podía llevar zapatos y que había mejorado mucho en cuanto al sueño. A las 12 semanas del tratamiento, el dolor seguía ausente y la movilidad de la enferma había aumentado mucho a pesar de cierto entumecimiento. Por desgracia, la paciente no estaba muy convencida de si someterse o no a nuevos tratamientos a pesar del éxito obtenido.

A raíz de este caso, el tratamiento con el parche de capsaicina al $8 \%$ se percibió de tres formas distintas. En primer lugar, su oncólogo llegó a la conclusión de que el tratamiento no había funcionado porque la paciente se quejaba de lo doloroso que había sido, sin mencionar la analgesia que había logrado. En segundo lugar, el médico generalista que vio a la paciente dos veces después del tratamiento ni se enteró del éxito que había obtenido la aplicación del parche de capsaicina al 8\%. En tercer lugar, el marido de la paciente no sabía que esta ya no tenía dolor hasta que este punto se aclaró de manera específica durante la consulta de seguimiento al cabo de 12 semanas, a la que asistió. Ninguno de ellos se dio cuenta de que la paciente ya podía calzarse. En general, el mensaje de que el parche de capsaicina al 8\% había sido eficaz, consiguiendo una analgesia prolongada, mejorando el sueño y la movilidad, y permitiendo el uso de calzado, acabó perdiéndose debido a las molestias del tratamiento en sí. Para evitar este problema y mejorar la experiencia vivida por las pacientes, debe avisarse de dolor quemante de inicio diferido. Los pacientes deben conocer perfectamente que es probable que tengan molestias, el momento en que podrían comenzar, el tiempo que podrían durar y la manera de tratarlas mediante bolsas de frío, analgésicos orales y la continuación de la medicación analgésica en curso. Además, los médicos referidores de estos pacientes, deben conocer los beneficios del tratamiento con el parche de capsaicina al $8 \%$, es decir, la analgesia que produce y la mejoría funcional que consigue. Entre los aspectos importantes a tener en cuenta al tratar pacientes con el parche de capsaicina al $8 \%$ están la forma óptima de atenderles después de la aplicación, especialmente durante la primera semana. Los médicos que usan el parche de capsaicina al $8 \%$ también deben plantearse el mensaje que envían a los otros profesionales de la salud.

El Dr. Bhaskar preguntó a los delegados qué método les parecía el más eficaz para lograr la analgesia después del tratamiento. La mayoría de los delegados dijeron que el frío era lo mejor. De las 37 respuestas, 22 se referían a alguna forma de aplicar frío (Fig. 5).

Tan solo un pequeño número de asistentes habían conocido pacientes con molestias extremas a causa del tratamiento. En la experiencia del Dr. Bhaskar, los pacientes con mezcla de dolor y entumecimiento son los que sienten menos dolor, mientras que quienes presentan alodinia sufren más en los primeros días posteriores al tratamiento.

\section{Coloquio}

- ¿Cuál es el número máximo de parches que se pueden usar en una sola aplicación? Según la ficha técnica se deben usar un máximo de cuatro parches a la vez. Sin embargo, si se necesitan más, por ejemplo otro medio parche, este se usaría en la misma aplicación. Esta práctica se realiza en la clínica del Dr. Bhaskar pero no está recomendada por Astellas.

- ¿Es posible que la causa de la reducción del dolor fuera la suspensión de la quimioterapia? No en este caso. Con algunos quimioterápicos, el dolor disminuye al suspender el tratamiento. Sin embargo, con el oxaliplatino, el dolor persiste y a menudo empeora 


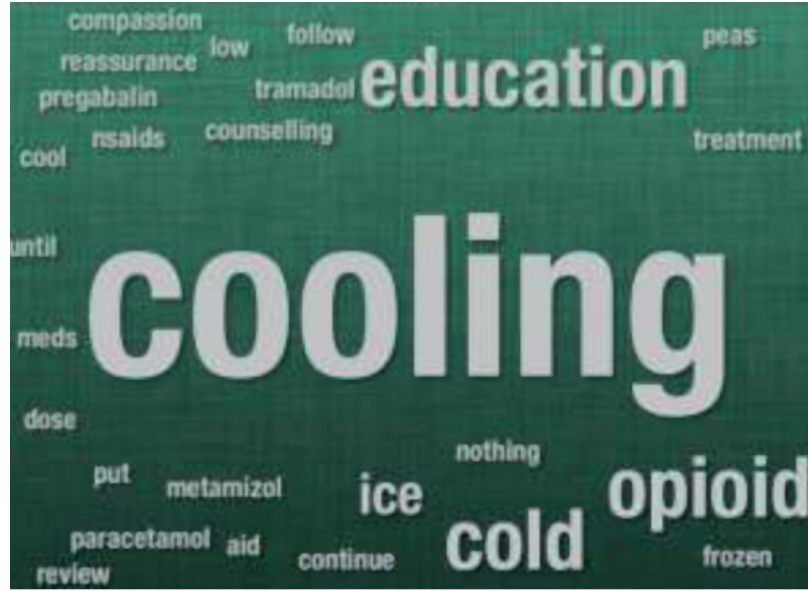

Fig. 5. Según su experiencia, ¿cuál es el método más eficaz de analgesia para después del tratamiento? $\mathrm{N}=25$ (tan solo 25 respuestas se hallaban debajo de la pregunta correcta y pudieron incluirse dentro de la nube de palabras, pero hubo 37 respuestas en total).

al suspender la quimioterapia, y esta paciente en concreto tuvo dolor durante mucho tiempo después de la retirada y refería que el dolor iba a peor.

- ¿Qué pasaría si un paciente acude con neuropatía bilateral de la mano o el pie? ¿Cómo abordaría el tratamiento de este paciente? Le preguntaría qué zona le gustaría tratar primero: las manos o los pies. No traten las manos y los pies en una misma sesión; pídanle al paciente que vuelva para tratarse la otra zona. No esperen 3 meses antes de tratar el resto.

- ¿Existen factores capaces de predecir la respuesta al tratamiento como, p. ej., la alodinia? Los pacientes con entumecimiento solamente no se tratarían con el parche de capsaicina porque sería poco probable que respondieran, pero los pacientes con entumecimiento y dolor sí se tratarían. En nuestra experiencia, los pacientes con signos positivos consiguen resultados mejores.

- ¿Cuántos pacientes trata al mismo tiempo? Normalmente se tratan tres pacientes en una sola sesión pero, si todos ellos precisan tratamiento de los pies, entonces se tratan solo dos. Se tarda más tiempo en cortar y aplicar el parche si se trata de los pies.

\section{EL PARCHE DE CAPSAICINA AL 8\% COMO TRATAMIENTO EFICAZ DEL DOLOR NEUROPÁTICO POSTOPERATORIO DESPUÉS DE VARIAS OPERACIONES DE CADERA EN UNA PACIENTE CON MÚLTIPLES PROCESOS COMÓRBIDOS}

El Dr. Hanno Jaeger presentó el caso de una paciente que tenía DN postoperatorio después de someterse a

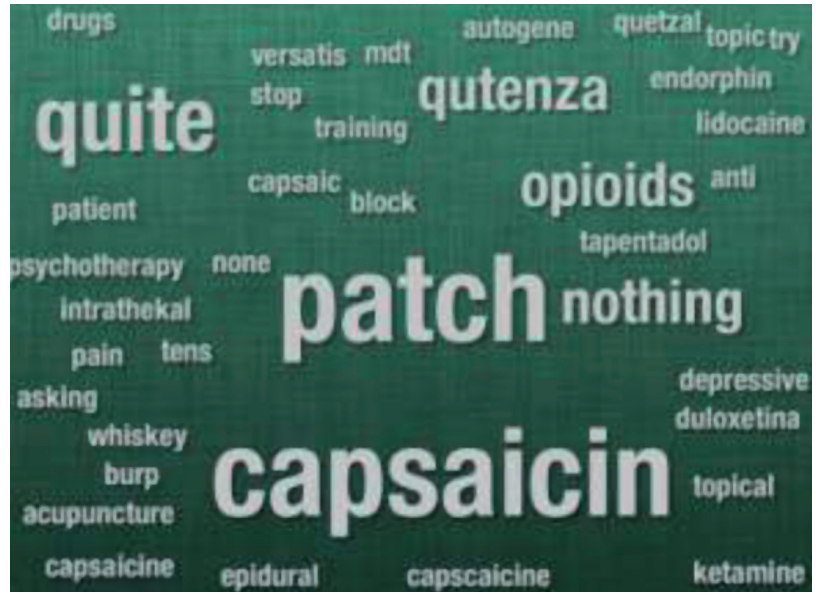

Fig. 6. ¿Qué añadirían a la medicación analgésica? $\mathrm{N}=34$.

operaciones de cadera y que también presentaba comorbilidad múltiple. Esta mujer de 74 años tenía epilepsia familiar, hipertensión, depresión, hiperlipoproteinemia, cardiopatía coronaria y cirugías asociadas, hernias, estenosis uretral, congestión renal, aneurisma y osteoporosis. La paciente tenía una lesión nerviosa a raíz de una operación de varios ganglios en 1950 y se había fracturado el cuello del fémur en 2002. Después de esto, se le retiró una placa en agosto de 2002 por haberse fracturado y se le puso una endoprótesis. Se le prolongó el vástago en agosto de 2003 y se le cambió en abril de 2009 por aflojamiento a causa de osteoporosis. La puntuación de dolor era una NRS de 6-9, el dolor era constante con exacerbaciones y de tipo punzante y lancinante. El dolor se desencadenaba al caminar, con el roce y con el agua fría. Tomaba diversos medicamentos para la patología cardiovascular y también había probado con pregabalina, gabapentina, lamotrigina y parches de fentanilo para aliviar el dolor. Todos se suspendieron por ineficaces o por no poder tolerarlos.

El Dr. Jaeger preguntó a los participantes qué analgésico añadirían a la pauta de tratamiento y 12/34 respondieron que capsaicina o Qutenza (Fig. 6).

El tratamiento añadido en la realidad había sido de hidromorfona y metamizol antes del parche de capsaicina al $8 \%$. La paciente recibió tratamiento en una amplia zona del muslo. Después del tratamiento, la cara lateral del muslo seguía siendo blanca mientas que la medial estaba eritematosa (Fig. 7).

El Dr. Jaeger pidió a los participantes que dijeran si creían o no que el tratamiento había funcionado y la mitad de los grupos pensaban que había tenido éxito al 50\% (Tabla VII). Esto es correcto; el tratamiento con el parche de capasaicina al $8 \%$ alivió el dolor en un $50 \%$ en esta paciente. 


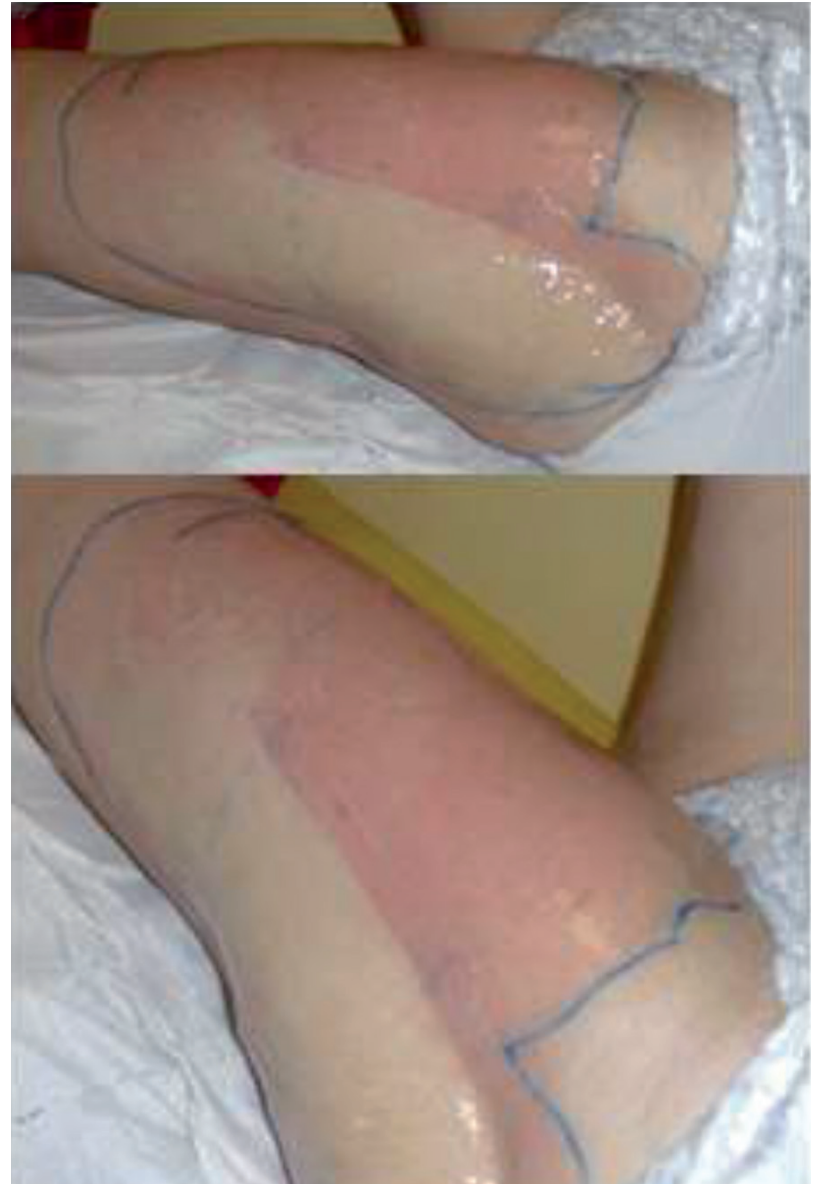

Fig. 7. Zonas reactiva y tratada. Solo la parte medial del muslo tenía eritema después del tratamiento con dosis altas de capsaicina.
TABLA VII. ¿CÓMO DE BIEN PIENSAN QUE FUNCIONÓ EL TRATAMIENTO? $\mathrm{N}=28$.

\begin{tabular}{ll}
$0 \%$ & $0 \%$ \\
$25 \%$ & $21 \%$ \\
$50 \%$ & $50 \%$ \\
$100 \%$ & $29 \%$ \\
\hline
\end{tabular}

\section{Coloquio}

- ¿Por qué parece funcionar bien Qutenza en los pacientes con DN postoperatorio? Como con las demás lesiones nerviosas, en la zona del DN postoperatorio hay expresión de receptores TRPV1. Probablemente, el tratamiento con el parche de capsaicina al $8 \%$ actúa sobre la sensibilización central.

- ¿Hace algo en particular para tratar de aumentar la respuesta al tratamiento? La medicación concomitante no se reduce inmediatamente después del tratamiento para evitar que los pacientes se sientan peor; al cabo de 3-4 semanas se comentan los efectos con los pacientes y se decide la reducción gradual de los opioides o los antiepilépticos.

- ¿Con qué frecuencia sigue a los pacientes tras el tratamiento? Se contacta con los pacientes, 24 horas después del tratamiento y después por teléfono pasadas 2-3 semanas. 